\title{
Pretreatment of rapid detection of veterinary drug residue in eggs
}

\author{
Xin Wang, Qigen Tong \\ Beijing University of Agriculture \\ Beijing 102206, China \\ e-mail: 2314213675@qq.com
}

\begin{abstract}
Eggs in the standard method for rapid detection of veterinary drug residue has not been formed, therefore, it is necessary to explore a rapid, highly effective, the total amount and qualitative method of rapid detection of antibiotic residues in eggs. Many different kinds of veterinary drugs on the market, this study selected: tetracycline, beta lactam, sulfa, fluoroquinolone for veterinary drug residue detection pretreatment research, finally to strip test results prior to determine the best treatment plan.
\end{abstract}

Keywords-veterinary drug residue; pretreatment; rapid detection

\section{INTRODUCTION}

Veterinary drug residue is refers to the accumulation of livestock animal medication after or stays in the body or prototype drugs in animal products such as eggs or their metabolites, including related to the veterinary drug residue[1]. These drugs can enter the human food chain through transfer, enrichment of the animal body, eventually leading to human in the process of eating the accumulation of veterinary drugs. Control of veterinary drug residues in eggs plays an important role in human life and health. Veterinary drug residue detection is an important means of eggs food safety, the rapid detection of standard has not been formed and its preparation method, therefore the experiment was carried out to explore, use is made of the existing market the rapid detection of veterinary drug residue detection milk strip for eggs of veterinary drug residues detection, the need for a series of eggs before treatment, including removal of the fat, to protein and so on. In theory, many different kinds of veterinary drug residue in eggs, this study selected: tetracycline, beta lactam, sulfa, fluoroquinolone for veterinary drug residue detection research.

Control of veterinary drug residues in eggs plays an important role in human life and health. Veterinary drug residue detection is an important means of eggs food safety, the existing detection methods at home and abroad for capillary electrophoresis, fluorescence method, uv spectrophotometry, chemiluminescence method, atomic absorption spectrophotometry, microbiological method, high performance liquid chromatography (HPLC) method and liquid mass usage, etc, but has not been a rapid detection of veterinary drug residue in eggs standard method, therefore, it is necessary to explore a rapid, highly effective, the total amount and qualitative method of rapid detection of antibiotic residues in eggs. There are many kinds of veterinary drugs on the market, this study selected: tetracycline, beta lactam, sulfa, fluoroquinolone for veterinary drug residue detection research

\section{MATERIALS AND METHODS}

Eggs, northern suburb of Beijing changping farmers market to buy; The rapid detection of strip, Beijing often state biological technology co., LTD.; Organic reagents, analytical pure, west long chemical co., LTD.

\section{EXPERIMENT}

\section{A. Tetracycline class antibiotic residues detection of egg sample pretreatment}

Chloramphenicol, oxytetracycline, tetracycline for common three kinds of tetracycline antibiotics. Tetracycline class of antibiotics, tetracyclines, TCs) is yellow crystalline powder, taste bitter, solubility in the alcohol (such as methanol and ethanol), such as ethyl acetate, acetone, acetonitrile dissolved in organic solvent is smaller. This kind of antibiotic produced by Streptomyces, on the chemical structure are hydrogenated pian and four benzene derivatives (pictured) basic structure, near the visible area near $(350 \mathrm{~nm})$ with strong ultraviolet absorption. Composition is complicated in biological samples, interference of tetracycline class of antibiotics on material is numerous, high concentration of salt in the sample and the existence of large molecular weight proteins will seriously affect the rapid detection of tetracycline class of antibiotics, so the pretreatment of the sample become one of the most important step in the sample analysis, adopt the method of reasonable and effective will tetracycline antibiotics extracted from the matrix of complex biological samples and to purify is particularly important.

By tetracycline compounds in weak acid solution is stable, so often need to extract the target under the condition of weak acid composition, molecular weight protein precipitation and biological sample cuhk, generally USES the extraction solvents including citric acid salt, phosphate, trichloroacetic acid and perchloric acid, etc [2].

Weigh and homogenizer is homogeneous whole egg sample in $5 \mathrm{~g}$, put in $50 \mathrm{~mL}$ with plug polypropylene centrifugal tube, add $5,8,10,15,20 \mathrm{ml} 0.1 \mathrm{~mol} / \mathrm{L}$ citrate, vortex mixing $1 \mathrm{~min}$, oscillation 5, 10, 15, $20 \mathrm{~min}, 10000$ $\mathrm{r} / \mathrm{min}$ centrifuge for $10 \mathrm{~min}$, the supernatant fluid to transfer to another $50 \mathrm{~mL}$ centrifuge tube, the residue with $5,8,10,15,20 \mathrm{~mL} 0.1 \mathrm{~mol} / \mathrm{L}$ citrate repeat extraction twice, clear liquid on merger, shake well, and let stand for $10 \mathrm{~min}$, after $0.22 \mu$ morganic membrane on rapid test. 


\section{B. Beta lactam class residue detection of egg sample pretreatment}

Aminoglycoside antibiotics will act as a veterinary drug used to treat meat and dairy animals, so we need an analysis of the residual effective analysis method in these goods. This class of antibiotics for residue analysis put forward a huge challenge. Unlike most other antibiotics, these compounds could not using acetonitrile, or other organic solvent extracted from the organization or dairy products. In this study, with trichloroacetic acid (TCA) aqueous buffer will aminoglycoside antibiotics extracted from meat or dairy products. Join the TCA can make protein precipitation and inhibit protein with the analyte. Before the LC/MS analysis, the use of effective solid phase extraction (SPE) purification operation to remove residual TCA, to minimize the total extract interference. Through the use of the Oasis HLB (efficient, water can be invasive inverting absorber), in the milk and meat can obtain good solid phase extraction yield and purification effect.Ampicillin, the free acid containing 3 molecular crystal water. White crystalline powder. Taste slightly bitter. Slightly soluble in water, insoluble in ethanol, dissolve in the dilute acid dilute alkali solution. Soluble in water, soluble in ethanol. [3].

Ampicillin and ampicillin. The free acid containing 3 molecular crystal water (for internal use); White crystalline powder. Taste slightly bitter. Slightly soluble in water, insoluble in ethanol, dissolve in the dilute acid dilute alkali solution. $\mathrm{PKa}$ of 2.5 and 7.3. $0.25 \%$ aqueous solution of $\mathrm{pH} 3.5 \sim 5$. 5. The structural formula below. Salt, the sodium for injection is white or kind of white powder or crystal. Odourless or slight odor, taste bitter. Have led to wet. Soluble in water, slightly soluble in ethanol. $10 \%$ aqueous solution of $\mathrm{pH}$ for $8 \sim 10$. Xudong will whole egg, egg white and yolk homogenate respectively, accurately say $5 \mathrm{~g}$ and grout, put in $50 \mathrm{~mL}$ with centrifugal pipe plug, add $5 \mathrm{~mL}$ of acetonitrile solution set spiral mixer blender $1 \mathrm{~min}$, then add $15 \mathrm{~mL}$ of acetonitrile solution, the swirling disaster $2 \mathrm{~min}$, oscillation and mixed with $8000 \mathrm{xg}$ centrifuge for $10 \mathrm{~min}$, the temperature is set to $10{ }^{\circ} \mathrm{C}$. Will clear liquid transferred to another $50 \mathrm{~mL}$ plug in the centrifuge tube, to add $20 \mathrm{~mL}$ with ultrapure water saturated me curse the methylene chloride solution blending 2 min (drug dissolved in the upper water, acetonitrile, soluble in methylene chloride solution

Yan-hong song said samples from $2.0 \mathrm{~g}$ homogenate of egg yolk and egg white, accurate to $0.1 \mathrm{~g}$, put in $50 \mathrm{ml}$ with centrifugal pipe plug, add $10 \mathrm{ml}$ of acetonitrile, intense blend for 5 minutes on the vortex mixer, oscillator with low speed oscillation omin, 3 to $5400 \mathrm{r} / \mathrm{min}$, the centrifugal 10 minutes, supernatant fluid in a centrifuge tube, and add $8 \mathrm{ml}$ of acetonitrile to slag, repeat extraction time, clear liquid on merger, $35{ }^{\circ} \mathrm{C}$ water bath nitrogen blow dry. In $2 \mathrm{ml}$ (mobile phase of methanol/1 \% ice acetic acid solution: $23 / 77, \mathrm{~V} / \mathrm{V}$ ) dissolve yolk residue, add $3 \mathrm{ml} \mathrm{n}$-hexane, oscillation let stand for 5 minutes, 10 minutes at $3000 \mathrm{r} / \mathrm{min}$, the centrifugal, remove $\mathrm{n}$-hexane layer through a straw, add the $3 \mathrm{ml} \mathrm{n}$-hexane repeat to fat, $0.22 \mathrm{mu} \mathrm{m}$ organic membrane, for the liquid chromatographic; Egg white without removal of the fat, with LML mobile phase (1\% methanol/ice acetic acid solution: 28/72, V/V) dissolved residue, after $0.22 \mathrm{mu} \mathrm{m}$ membrane filter, the liquid chromatograph.

According to take in the homogenizer is homogeneous whole egg sample $2 \mathrm{~g}$, put in $50 \mathrm{ml}$ with centrifugal pipe plug, add $5,10,15,20 \mathrm{ml}$ of acetonitrile, intense mixing 5 min on the vortex mixer, oscillator with low speed oscillation, $10,15,20,25 \mathrm{~min}$ to $5400 \mathrm{r} / \mathrm{min}$, the centrifugal $10 \mathrm{~min}$, supernatant fluid in a centrifuge tube, and add $8 \mathrm{ml}$ of acetonitrile to slag, repeat extraction time, clear liquid on merger, $35^{\circ} \mathrm{C}$ water bath nitrogen blow dry. Add $3 \mathrm{ml} \mathrm{n}$-hexane, oscillation let stand for 5 minutes, 10 minutes at $3000 \mathrm{r} / \mathrm{min}$, the centrifugal, remove $\mathrm{n}$ hexane layer through a straw, add the $3 \mathrm{ml} \mathrm{n}$-hexane repeat to fat, after $0.22 \mu \mathrm{m}$ organic membrane on rapid test.

Sulfa drugs, its molecular weight between $170 \sim 300$, soluble in water, soluble in ethanol and acetone, almost insoluble in chloroform and ether. Except for alkaline sulfaguanidine, sulfa drugs because of containing the primary amine and sulfonamide based in acid and alkali sex, soluble in acid and alkali solution. Acid is weak and easy to absorb carbon dioxide from the air and carbonate precipitation. Because of its structure with a benzene ring, all have the ultraviolet absorption [4]. Add egg samples samples from $2 \mathrm{~g}$ to $5,10,15,20 \mathrm{ml}$ ethyl acetate oscillating up and down 5, 10, 15, $20 \mathrm{~min}$, at room temperature to $5000 \mathrm{r} / \mathrm{min}$ centrifugal $5 \mathrm{~min}$, take the lower liquid, after $0.22 \mathrm{mu} \mathrm{m}$ organic membrane on rapid immunochromatographic test. According to the article late rapid test results, it is concluded that under the condition of the following the fastest the most accurate test result. Add egg samples from $2 \mathrm{~g}$ to $5 \mathrm{ml} 5 \mathrm{~min}$, ethyl acetate and oscillations at room temperature to $5000 \mathrm{r} / \mathrm{min}$ centrifugal $5 \mathrm{~min}$, take the lower liquid, after $0.22 \mu \mathrm{m}$ organic membrane on rapid test.

\section{Fluoroquinolone residues detection of egg sample pretreatment}

Fluoroquinolone drugs (FQs) are white or light yellow crystal powder, usually free acid soluble in dilute alkali and glacial acetic acid, dilute acid solution, the water in the pH6 $\sim 8$ solubility, minimum in methanol, chloroform, ether and most difficult soluble or insoluble in the solvent. The structure of benzene and heterocyclic or heterocyclic skeleton and carbonyl and carboxyl, heteroatom such as chromophore or help base group of conjugate system, in the ultraviolet region characteristic and strong absorption, the ultraviolet spectrum contains several absorption peak: $240 \sim 300 \mathrm{~nm} .330 \sim 350 \mathrm{~nm}$. FQs itself also have fluorescent properties, the $\mathrm{Ex}=280 \sim 330 \mathrm{~nm}$, Em $=410 \sim 430 \mathrm{~nm}$. FQs acid-base amphoteric compounds, ultraviolet absorption spectrum in different $\mathrm{pH}$ medium will be slightly different [5].

In fresh egg, after the break with low speed homogenizer homogeneous egg samples, egg white and yellow when fully mixing, as the blank sample. After taking homogenate of blank sample, add the appropriate concentration of standard solution as the blank to add sample. In $2.0 \mathrm{~g}$ egg samples to $15 \mathrm{ml}$ polystyrene in centrifuge tube, add 5, 10, 15, $20 \mathrm{ml}$ of acetonitrile, fully use oscillator oscillation $5 \mathrm{~min}$. Learned that $2 \mathrm{ml}$ supernatant fluid to $10 \mathrm{ml}$ of clean glass tube, 50 to $60{ }^{\circ} \mathrm{C}$ dry nitrogen flow. Add 2, 5, 8, $10 \mathrm{ml} \mathrm{n}$-hexane, using vortex finder vortex $1 \mathrm{~min}$, centrifugal $5 \mathrm{~min}$ at room 
temperature, remove the upper organic phase, take $50 \mu \mathrm{l}$ for analysis. According to the article late rapid immunochromatographic test results, it is concluded that under the condition of the following the fastest the most accurate test result. In $2.0 \mathrm{~g}$ egg samples to $15 \mathrm{ml}$ polystyrene in centrifuge tube, add $5 \mathrm{ml}$ of acetonitrile, fully use oscillator oscillation $5 \mathrm{~min}$. Learned that $2 \mathrm{ml}$ supernatant fluid to $10 \mathrm{ml}$ of clean glass tube, 50 to $60{ }^{\circ} \mathrm{C}$ dry nitrogen flow. Add $5 \mathrm{ml} \mathrm{n}$-hexane, using vortex finder vortex $1 \mathrm{~min}$.

\section{Sulfonamides residues detection of egg sample pretreatment}

Sulfa drugs, its molecular weight between $170 \sim 300$, its chemical structure is as follows. Slightly soluble in water, soluble in ethanol and acetone, almost insoluble in chloroform and ether. Except for alkaline sulfaguanidine, sulfa drugs because of containing the primary amine and sulfonamide based in acid and alkali sex, soluble in acid and alkali solution. Most pKa sulfa drugs within $5 \sim 8$, isoelectric point for $3 \sim 5$, a few pKa is $8.5 \sim 10.5$. Acid is weak and easy to absorb carbon dioxide from the air and carbonate precipitation precipitation. Because of its structure with a benzene ring, so all have the ultraviolet absorption. Zhong Ziqing said in $5 \mathrm{~g}$ egg samples, accurate to $0.01 \mathrm{~g}, 10$ copies of put in $50 \mathrm{ml}$ centrifuge tube. Each addition concentration set A, B, C three parallel, each sample set A blank control. Add quantity respectively for $10,100,200 \mathrm{ng} / \mathrm{g}$. Add the sample after $30 \mathrm{~min}$. Each centrifugal pipe add $20 \mathrm{~g}$ anhydrous sodium sulfate and $20 \mathrm{ml}$ of acetonitrile, homogeneous $2 \mathrm{~min}$, to $3000 \mathrm{r} / \mathrm{min}$, the centrifugal $3 \mathrm{~min}$.

Supernatant fluid in $100 \mathrm{ml}$ bottle heart, residue add $20 \mathrm{ml}$ of acetonitrile, repeat the above for 1 times. Merge extract, to the heart in a bottle and add $10 \mathrm{ml}$ isopropyl alcohol, using rotary evaporator in $50{ }^{\circ} \mathrm{C}$ water bath to dry, accurate flow with $1 \mathrm{ml}$ and $1 \mathrm{ml} \mathrm{n}$-hexane soluble residues. Transferred to the $5 \mathrm{ml}$ centrifuge tube, the vortex $1 \mathrm{~min}, 3000 \mathrm{r} / \mathrm{min}$, the centrifugal $3 \mathrm{~min}$, abandon to upper n-hexane, add $1 \mathrm{ml} \mathrm{n}$-hexane, repeat the above steps. Take the lower solution, after $0.2 \mathrm{mu}$ membrane filter, using liquid chromatography - tandem mass spectrometry determination. Add egg samples samples from $2 \mathrm{~g}$ to $4 \mathrm{ml}$ ethyl acetate oscillation for 3 minutes, up and down at $5000 \mathrm{r} / \mathrm{min}$, the centrifugal 5 minutes, take $300 \mathrm{mu} 1$ upper liquid $80^{\circ} \mathrm{C}$ water bath top up or down in nitrogen blow dry, with $150 \mathrm{mu} 10.01 \mathrm{~mol} / 1 \mathrm{pH} 7.4$ slightly dissolve the residue of phosphate buffer.

\section{RESULTS AND ANALYSIS}

\section{A. Ampicillin, tetracycline class antibiotics residue sample detection}

Beta lactam and tetracycline class rapid test strip applied the principle of the competitive inhibition of immune chromatography samples in the beta lactam classes and tetracycline drugs in the process of flow and colloidal gold marked specificity monoclonal antibody, suppresses the antibodies and lines of NC membrane (B, $\mathrm{T}$ line) on the beta lactam classes and tetracycline drugs a combination of BSA coupling, leading to lines of depth changes. When the samples have no beta lactam classes and tetracycline drugs or beta lactam and tetracycline drugs concentrations below the detection limit, $\mathrm{B}, \mathrm{T}$ line color; When the samples in the beta lactam classes and tetracycline drugs concentration equal to or higher than the detection limit, B, T line color; And no matter whether the sample contains beta lactam type and tetracycline drugs, color quality control line $\mathrm{C}$ line, to test effectively.

Sample preparation extraction buffer (Na2EDTA NH4OOCH3 $10 \mathrm{mM} / 0.4 \mathrm{mM} / 1 \% \mathrm{NaCl} / 2 \%$ TCA) : $0.77 \mathrm{~g}$ ammonium acetate (NH4OOCH3) in 11 of the volumetric flask. Add about $900 \mathrm{~mL}$ of reagent water, dissolve. Use $1 \mathrm{~N} 1 \mathrm{~N} \mathrm{HCl}$ or $\mathrm{NaOH}$ to adjust $\mathrm{pH}$ value to 4.0. Add $0.15 \mathrm{~g}$ ethylenediamine tetraacetic acid disodium (Na2EDTA. 2 h2o), $5 \mathrm{~g}$ of sodium chloride $(\mathrm{NaCl})$ and 20 $\mathrm{g}$ trifluoroacetic acid (TCA). Mix, make the solid solution, add reagent water to scale. Preliminary extraction: $2 \mathrm{~g}$ even cattle tissues or $10 \mathrm{~mL}$ milk in $50 \mathrm{~mL}$ centrifuge tube. Add $20 \mathrm{~mL}$ extraction buffer, vortex 10 seconds, and then fully shake the 1 minute. Sample of the centrifugal 5 minutes under 4000 RPM, clear fluid collection. According to the need to use dilute $\mathrm{HCl}$ or $\mathrm{NaOH}$ will clear liquid $\mathrm{pH}$ adjustment to $6.5+/-0.5$.

\section{B. Fluoroquinolone, sulfonamides residue sample detection}

Sulfa and fluoroquinolone combined rapid test strip applied the principle of the competitive inhibition of immune chromatography samples of sulfa drugs and fluoroquinolone drugs in the process of flow combined with colloidal gold marked specificity monoclonal antibody, suppresses the antibodies and lines of NC membrane (T) on the sulfa and fluoroquinolone - a combination of BSA coupling, resulting in lines of color depth changes. When there is no sample of sulfa drugs and fluoroquinolone drugs or sulfa drugs and fluoroquinolone drugs concentrations below the detection limit, T1, T2 line color; When sulfa drug concentration in the sample is equal to or higher than the detection limit, a T1 line color, T2 line color; When the sample of fluoroquinolone drugs concentration equal to or higher than the detection limit, T2 line color, not a T1 line color; And no matter whether samples containing sulfa drugs and fluoroquinolone drugs, quality control line (C) all can color, to show detection effectively.

Marry remove all reagent from cold storage environment, balance at room temperature $\left(25^{\circ} \mathrm{C}\right) 20-30$ min rapidly. The kits and standard sample of the corresponding microporous serial number, each sample and standard substance do two parallel holes. Add to the corresponding standard/sample $50 \mathrm{mu} 1$ microporous, soon to join enzyme mark two $50 \mathrm{mu} 1 /$ hole, oscillation blending, $25^{\circ} \mathrm{C}$ avoid light reaction after $60 \mathrm{~min}$ in the environment with washing liquid is $250 \mathrm{mu} 1 /$ hole, full of washing 4-5 times. Join the substrate liquid A liquid 50 $\mathrm{mu} 1 /$ hole, then add the substrate liquid B liquid $50 \mathrm{mu}$ 1/hole, oscillation and mixed $25{ }^{\circ} \mathrm{C}$ avoid light environment reaction after $30 \mathrm{~min}$. Add terminated liquid $50 \mathrm{mu}$ 1/hole, blending, enzyme standard instrument set in each hole OD value at $450 \mathrm{~nm}$. To standard percentage absorbance (samples/zero absorbance value of standard absorbance value) as the ordinate, standard concentration (PPB), the abscissa denotes the semilog, draw standard curve. The percentage of sample absorbance generation into the standard curve, read the concentration of the sample corresponds to the standard curve, multiplied by 
the corresponding dilution ratio. SPE purification: this study used the Oasis HLB 96 - well plates $(30 \mathrm{mg})$. If necessary, can use a $1 \mathrm{cc} / 30 \mathrm{mg}$ extraction column. Successively used $1.5 \mathrm{~mL}$ of methanol, $1.5 \mathrm{~mL}$ water, balance board hole or extraction column. The flow velocity is set to $1 \mathrm{~mL} / \mathrm{min}$ or less. Join the preliminary extraction to get supernatant after $\mathrm{pH}$ adjustment, for tissue samples, add $1 \mathrm{~mL}$ solution, samples for dairy products, to join a $1.5 \mathrm{~mL}$ solution. $1 \mathrm{~mL}$ water to rinse. With 0.5 mL 10:5:8 5 formate/isopropanol/water elution. To join (including 1.5 L HFBA, using UPLC/MS/MS analysis[6].

\section{Sulfonamides residue sample detection}

Li Kui by using immune chromatography rapid determination of sulfonamides residues in eggs. From $4{ }^{\circ} \mathrm{C}$ strip out of the fridge, return to room temperature. Blank respectively $120 \mathrm{mu} 1$ sulfa drug standard samples and waiting for inspection, in turn, add to the MPP hole, insert the strip sample end microporous plate hole, make holes in the liquid by capillary action up swimming, reaction after 10 minutes, judge the results. Sulfa drugs standard strip blank lines appear red stripe; If waiting for the sample strip lines appear similar to the blank lines of standard red stripe, sulfa drugs, not detected in the samples that sentence to negative; Visible light on the blank lines of standard color light red stripe, description of sulfanilamide in oxygen pyrimidine (SMM), sulfanilamide for oxygen pyrimidine (SMD), sulfanilamide dimethyl oxygen pyrimidine (SDM) concentration in the $20 \sim 100 \mathrm{mu} \mathrm{g} / \mathrm{kg}$ range, or SDZ concentration within $40 \sim 160 \mathrm{mu} \mathrm{g} / \mathrm{kg}$, jailed for weakly positive; If the inspected samples without color strip line, SMM, SMD, concentration of SDM in the sample that is more than $100 \mathrm{mu}$

\section{PROSPECT}

Laying hens production in our country in the world, but more than $95 \%$ of the eggs are produced by farmers and small businesses, as a result of the limitation of capital, technology, management and other aspects of vicious competition and market prices, the price of eggs was far below the normal production cost. Feed producers as the pursuit of profit, and extensive use of cheap inferior raw material; In order to reduce disease risk, abuse of antibiotics; For high yield, and extensive use of synthetic hormones. Individual farmers to buy cheap feed, and the imperfection of the supervision strength, have an opportunity to make illegal activities. Resulting in a decline in egg quality and nutrient unbalance, pathogenic bacteria, antibiotics, hormones and pesticide residues exceeds bid badly. The use of veterinary drugs not science, not specification, led to the occurrence of drug residues. To prevent avian disease, in the case of uncertain etiology abuse of antibiotics, optional increasing dosage, dosage change, do not obey to take medicine.

All in all, First is looking at home and abroad research status, can include physical and chemical properties of the target component, harm, types of existing research methods, and the pros and cons of each method, the current methods of GB and components limited threshold. Research status in a certain extent, have the latest research results. Research focus, and the light spot and so on also can have certain embodiment. And in the veterinary drug detection, drugs, veterinary drugs can be divided into categories of methods summary (similar components of structure, a lot of time there may be a lot of consistency test method).

\section{ACKNOWLEDGEMENTS}

It is a project supported by Science and technology achievements transformation and industrialization Program of China ("Natural clean" egg production and promotion) (PZM2013-014207-000054). The corresponding author is Qigen Tong.

\section{REFERENCES}

[1] Zhang, $\mathrm{C}, \mathrm{H}$ The harm of veterinary drug residues in animal foods and monitor countermeasures . Journal of gansu agricultural,11,pp.50-50,2004.

[2] Shen.Y.J.\&Gao.Z.B.\&Sun.G.W., Tetracycline residues in animal food detection method. Journal of Shanghai, animal husbandry and veterinary communications,6,pp.56-57,2006.

[3] Chen.T.B. \& Deng.W.H,Lu,W,H., Capillary electrophoresis method to detect antibiotic residues in honey.Journal of chromatography,19(1), pp.91-93,2001.

[4] Zhong.Z.Q. \& Huang.Z.H. \&Lai.W.H., Sulfonamides residues in eggs extraction method .Journal of food science,33(6), pp.154158,2012 .

[5] Wang.X.Y \& Zhu.L.Q., Three kinds of fluoroquinolone drugs in chicken body tissue residues. Journal of food science, 28(11), pp.468-472,2008.

[6] Tavakoli, N., Varshosaz, J., Dorkoosh, F., \& Zargarzadeh, M. R., Development and validation of a simple HPLC method for simultaneous in vitro determination of amoxicillin and metronidazole at single wavelength. Journal of pharmaceutical and biomedical analysis, 43(1), pp.325-329.2007. 